\title{
On the shoulders of (real) giants
}

\author{
Ian D Pavord, ${ }^{1}$ Andrew Bush ${ }^{2}$
}

It is a daunting task to take over the reins of Thorax, after the fantastically successful last years. Thorax is ranked second in the world among respiratory journals, with an impact factor of 7.069 , and this is due mainly to the untiring efforts of the previous team, so ably headed by Wisia Wedzicha and Seb Johnston, building on the foundations laid by previous Editorial teams. It would be wrong not to acknowledge with respect and amazement the achievements to date. They have been the real giants on whose shoulders we stand (definitely not in the snide sense originally used by Newton). So we approach the next 5 years with some trepidation, but great excitement. Clearly we are not going to make radical changes; we want to continue to attract the best clinical work and basic science to the journal. We do believe there are areas on which we would like to focus to move the journal forward.

\section{RANDOMISED CONTROLLED TRIALS}

We want to facilitate the rapid publication of good clinical trials, the backbone of evidence-based medicine. We will offer peer review of the protocol when it has been registered on any approved site. If the protocol passes peer review, we will offer fast-track publication on completion of the trial, provided the protocol has been followed and, where appropriate, CONSORT requirements are met ${ }^{1}$ - these will be the sole criteria for publication. We will aim to have the trial published online within 2 weeks, with fast-tracking into the paper version. Furthermore, we will invite groups doing large trials to submit a paper describing their protocol as 'work in progress', to keep our community informed about new developments. ${ }^{2}$

\section{GUIDELINES}

The British Thoracic Society (BTS) guidelines have been important reading for

\footnotetext{
${ }^{1}$ Glenfield Hospital, UK; ${ }^{2}$ Imperial College \& Royal Brompton \& Harefield NHS Foundation Trust, UK

Correspondence to lan D Pavord, Department of Respiratory Medicine, Thoracic Surgery and Allergy, University Hospitals of Leicester NHS Trust, Glenfield Hospital, Groby Road, Leicester LE3 9QP, UK; ian.pavord@uhl-tr.nhs.uk
}

many of us, and these will continue to be published in Thorax. However, they can be (necessarily) very bulky. We will plan to publish an executive summary in the form of an editorial in the paper journal, with the full version published online, unless the expense of a supplement is felt to be justified.

\section{CHEST CLINICS}

We are both active practising clinicians with a research interest nearer the bedside than the bench. We are conscious that many readers without a major research interest would like to see more material of immediate clinical relevance. We plan to develop a new section in Thorax called 'Chest Clinics' to cater for this. This section will incorporate the very successful 'Images in Thorax' and a more condensed version of 'Lung Alerts', and include a new case-based review series, clinical cases for discussion, short reviews dealing with the clinical impact of new basic research findings, a section spotlighting the views and career highlights of distinguished colleagues who are retiring or nearing retirement and lighter pieces addressing aspects of clinical practice of relevance to the practising respiratory physician. We are happy to consider uncommisioned articles and suggestions for colleagues who should feature in our retirement series. Our aim is to increase the readability of the journal. We also hope to address educational needs by linking articles to a limited number of $\mathrm{MCO}$ questions.

\section{REVIEW ARTICLES}

Many people need to rely on high quality reviews to keep up to date. We will commission in two other areas. First, we will be asking our editorial team to produce high-quality reviews of the important papers published in Thorax and elsewhere over the previous 2 years. These will be critical and focused, and set in the context of previous work. Secondly, we feel the BTS Winter meeting deserves more attention. We will be asking members of the BTS Speciality Advisory Group to write reviews of the important work presented both in invited lectures and in abstracts at the meeting. Finally, we would like to receive proposals $(<500$ words) for state of the art review articles; these will be carefully considered and peer reviewed before an invitation to submit a full article is issued. All reviews will be peer reviewed to ensure good quality.

\section{CORRESPONDENCE COLUMNS}

We want to try to make this section more interactive. We will encourage short summary letters (200 words) arising from work published in the journal. The letter can have as long an online supplement as is needed (within reason). We will post all letters online (unless they are libellous or inaccurate) within 48 hours of receipt, and aim to publish the most significant comments in the paper version of the journal. There is the option just to submit a long version, but it is unlikely that we will publish long letters, unless of exceptional quality, in the paper version. The second area is case reports. We will no longer accept case reports as full papers, only as letters to the Editor. The maximum length for the paper copy will be 500 words; online supplements will be encouraged.

\section{THE REVIEW PROCESS}

We know that all authors want their papers accepted by return e-mail, with warm congratulations and no changes requested. This is not going to happen! We are committed to speeding up the review process. We will be streamlining the website for ease of access, and asking for a review with 3 weeks. Reviewers will get countdown e-alerts of the impending deadlines at regular intervals. We will be publicly acknowledging those reviewers who have looked at the most papers, and those who have returned the best and fastest reviews (but not yet naming and shaming the malcontents!). We will be reviewing the individual performances of all the Editorial team, including our own, in terms of turnaround time. We acknowledge that we owe it to our authors to give a timely and constructive response to their manuscripts.

\section{CONCLUSIONS}

We aim to build on the excellent work of our forbears. We believe we have recruited a very talented Editorial team. We will depend totally on the good will of the reviewers, and on you to submit the best manuscripts to us. We aim to publish exciting and clinically relevant work; to raise the impact factor, and snap at the heels of the 'Blue Journal'; and above all, to produce a journal that you all look forward to reading.

Competing interests None. 
Provenance and peer review Commissioned; not externally peer reviewed.

Thorax 2010;65:943-944.

doi:10.1136/thx.2010.147165

\section{REFERENCES}

1. Schulz KF, Altman DG, Moher D; for the CONSORT Group. CONSORT 2010 Statement: updated guidelines for reporting parallel group randomised trials. BMJ 2010;340:c332
2. Vestbo J; TORCH Study Group. The TORCH (TOwards a Revolution in COPD Health) survival study protocol. Eur Respir J 2004;24:206-10.

\section{Predicting mortality in the elderly with community-acquired pneumonia: should we design a new car or set a new 'speed limit'?}

\section{Veronica Brito, ${ }^{1}$ Michael S Niederman ${ }^{2}$}

Community-acquired pneumonia (CAP) is a common illness associated with increasing mortality rates that parallel the site of care. While outpatients have a risk of dying of $<5 \%$, those in hospital have a mortality rate of $12 \%$ and those managed in the ICU can have a chance of dying that exceeds $30 \%{ }^{1}$ In addition, delayed recognition of severe illness can add to mortality, with those admitted to the ICU late in the course of disease having a higher mortality than those admitted early. ${ }^{2}$ Thus, patient outcome is dependent on making an accurate assessment about where patients should be initially managed and the intensity of care that they should receive. To this end, a number of prognostic scoring systems have been developed for patients with CAP that can predict the mortality risk, which is then often applied as a surrogate for deciding the initial site of care..$^{1} 34$ Unfortunately, the risk of death is not always correlated with the need for a high level of care including need for ICU admission. In fact, patients who are young and otherwise healthy may benefit from ICU admission yet have a much lower predicted mortality risk than older patients with multiple medical comorbidities who may benefit little from

\footnotetext{
${ }^{1}$ Department of Pulmonary, Critical Care and Sleep Medicine, Winthrop University Hospital, Mineola, New York, USA; ${ }^{2}$ Department of Medicine, SUNY at Stony Brook, Chairman, Department of Medicine,

Winthrop-University Hospital, Mineola, New York, USA

Correspondence to Dr Michael S Niederman, Professor of Medicine, 222 Station Plaza N, Suite 509, Mineola, NY 11501, USA; mniederman@winthrop.org
}

admission to a hospital unit that provides a high level of care. Accurate definition of the site of care not only impacts mortality, but also the cost of care which rises incrementally from outpatient to inpatient to ICU management.

The most commonly used tools for predicting mortality-the CURB-65 (confusion, elevated blood urea nitrogen, elevated respiratory rate, low blood pressure and age $>65$ years), derived from the British Thoracic Society rule, ${ }^{5}$ and the Pneumonia Severity Index (PSI) ${ }^{1}$ - predict mortality by giving a point score to a number of acute and chronic disease variables, but both incorporate age into the scoring system. With CURB-65, age is a categorical variable ( $\geq$ or $<65$ years) while, with the PSI, it is a continuous linear variable. The implication from this approach is that age independently adds to the risk of death, but this is a complex issue since patients generally have more comorbid illnesses with advancing age and the independent contribution of age itself to mortality is uncertain. Kothe et $a l^{6}$ studied $>2000$ patients with CAP, $75 \%$ of whom were managed in hospital, and found that those aged $\geq 65$ years had a higher mortality than younger patients and that age was an independent risk factor for death, even after controlling for comorbid illness, severity of illness, site of residence (nursing home or not) and treatment-related factors. ${ }^{6}$ Similarly, Marrie et al, ${ }^{7}$ studying $>3000$ admitted patients with CAP, found that age was an independent mortality risk factor over and above the risk that could be attributed to disease severity using the PSI. ${ }^{7}$
Why should age be an independent risk factor for death from pneumonia? Elderly patients may present later in the course of illness than younger patients because the classic clinical symptoms of pneumonia are not always present, the vital sign parameters may be less abnormal than in younger patients, and both family members and physicians may initially overlook the diagnosis of pneumonia. In addition, even 'healthy ageing' may be associated with impairments in immunity and lung function, even in the absence of comorbid illness. Also, multiple chronic diseases are more prevalent in elderly patients than in younger patients, and it is possible that there is a synergy between multiple comorbidities in these older individuals that is not accounted for by prognostic scoring systems.

It is likely that existing scoring systems for CAP have limitations in patients with advancing age, but the extent of these limitations is unclear. The PSI was developed and validated as a way to identify patients with a low mortality risk who could be safely managed out of hospital, but it can potentially underestimate severity of illness, especially in young patients without comorbid illness who have acute abnormalities of vital signs, while overestimating the mortality risk in older patients with minimal acute disease processes but a high frequency of stable comorbid disease processes. Not all patients in a high PSI risk class need to be managed in the ICU. In a Spanish study of 457 patients with CAP in the highest mortality risk group (PSI class V), only 92 were admitted to the ICU. ${ }^{8}$ When patients were admitted to the ICU they tended to get more of their PSI points from acute rather than chronic illness, while the reverse was true for those patients in PSI risk class $\mathrm{V}$ who were not admitted to the ICU. On the other hand, a retrospective analysis comparing patients admitted to wards and to the ICU showed that, while the patients in the ICU had a higher PSI score than the ward patients, the cohort admitted to the ICU included patients in all PSI classes with 30\% falling into low PSI risk groups (I-III). ${ }^{9}$

The CURB-65 approach may be ideal for identifying patients with a high mortality risk because of acute vital sign 